\title{
Rating and Selection of Kinematic Linkages for Manipulators: Consideration of Parallelism and Number of Loops
}

\author{
A. Srinath ${ }^{*}, 1$ and A. Jagadeesh ${ }^{2}$ \\ ${ }^{I}$ Department of Mechanical Engineering, Koneru Lakshmaiah College of Engineering, Green Fields, Vaddeswaram, \\ Guntur District, A.P, India \\ ${ }^{2}$ Rungta College of Engineering and Technology, Bhilai, Chhatisgarh, India
}

\begin{abstract}
Choosing an optimum linkage for any robotic manipulator is an important task and also a tedious one as it needs to simulate/test all the linkages and finally compare them, as we are aware that there are numerous linkages available for any given purpose based on their input and output functions. A method is being proposed here which generates, simulates/tests the linkages; provided the type of input and output is fixed. This method shall yield the best possible linkage as it focuses on the structural as well as the functional aspects and has no limitation for any type of linkage.
\end{abstract}

Keywords: Parallelism, manipulator linkages, kinematic chains, simulations, optimum linkage.

\section{INTRODUCTION}

Planar Kinematic chains with d.o.f. (degree of freedom) $>1$ are considered for robotic application [1-6]. Unlike spatial linkages, a huge number of distinct planar chains are available; for example; nine-link 2 d.o.f of 40 and ten-link 3 d.o.f of 98 chains. All of them possess parallelism and the extent it exists in a chain is not known but is necessary for the designer in order to make a right choice of the structure.

The parallelism is of both structural and dimensional type $[7,8]$. It is understood that structural parallelism is influenced by the link dimensions i.e. variation of link dimensions will vary the parallelism and hence the rigidity. Thus it cannot be said which structure and what combination of link lengths will lead to optimum rigidity and lot of work is being done in this area. A simple measure is desirable to compare all the chains for the parallelism with specified link dimensions and this paper is an attempt in this direction.

\section{THEORY}

Consider a four-bar chain, (Fig. 1a), which is the simplest single d.o.f chain with structural parallelism among its links. Parallelism can be understood as the existence of two or more paths between links to transmit motion or power [7]. In the chain of Fig. (1a), link 3 receives motion from link 1 through links 2 and 4 . This is called structural parallelism. The above concept has coherence through graph theory. A chain can be converted into a graph which consists of vertices and edges. Every link of a chain is represented by a vertex while every joint is represented by an edge. With this understanding, the graph of the chain (Fig. 1a) can be drawn as shown in Fig. (1b).

*Address correspondence to this author at the Department of Mechanical Engineering, Koneru Lakshmaiah College of Engineering, Green Fields, Vaddeswaram, Guntur District, A.P, India; Tel: 091-8645-246948; Fax: 091-8645-247249;

E-mails: srinath_me@klce.ac.in, srichakra_atluri@rediffmail.com
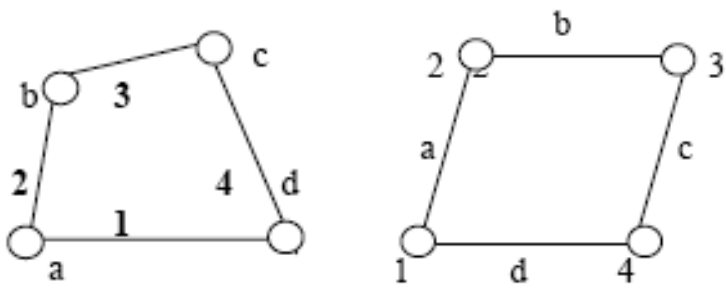

Fig. (1).

Links or vertices 1 and 3, for example are separated by two edges. Edges 1-2 and 2-3 constitute one path between links 1 and 3 while the edges 1-4 and 4-3 constitute another path. Thus, vertices 1 and 3 are parallely connected.

Now let us consider the same chain, of Fig. (1a) when all its links are equal in length, as shown in Fig. (2a) which is a parallelogram. This is called as dimensional parallelism. It should be noted that structural parallelism is a prerequisite to dimensional parallelism. It is evident that when link-1 is fixed and link-2 is driven with an angular velocity $\omega$, the angular velocity of link-4 will also be the same, while link-3 will have zero angular velocity.

From the above it can observed that

1) Incase of dimensionally parallel mechanisms the velocities and the accelerations of the parallel links will be identical.

2) As structural parallelism is a prerequisite to dimensional parallelism; in a chain with structural parallelism but having no dimensional parallelism; there will not be great variation in the velocities and accelerations of parallel links.

3) Parallel chains are more rigid.

4) If the input and output links do not differ much with respect to the ground link (self) from the view point of extent of parallelism, the displacement of the output link will be of the same order as that of the input link. 


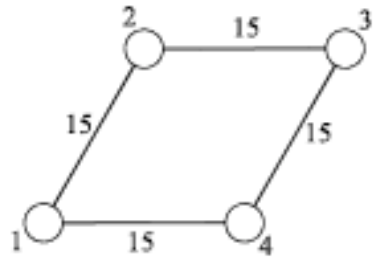

(a)

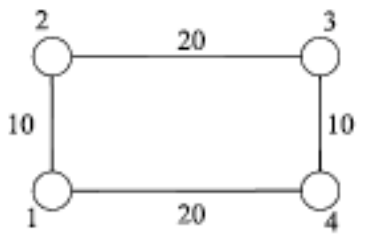

(b)

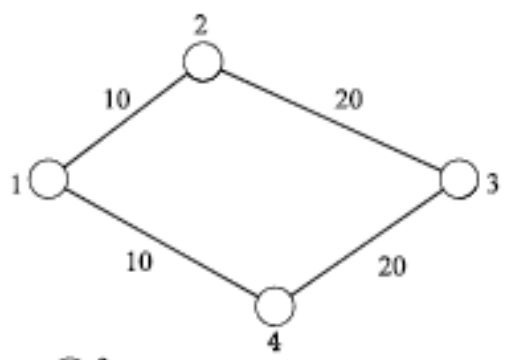

(c)

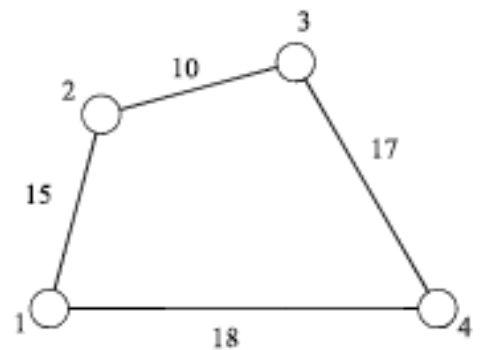

(d)

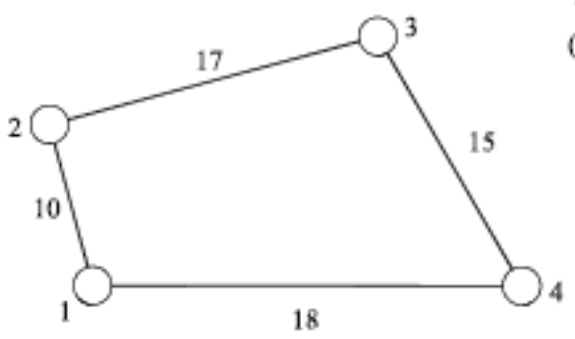

(e)

Fig. (2).

The above observations can be understood as below:

a) Parallel robots will have lesser work space.

b) In case of function generators, if the output link is less parallel than the input link with respect to the ground link, the displacement of the output link between every precision point will be higher than the corresponding input displacements. Non-linear functions have this characteristic and as such generation of non-linear functions will be more accurate in case of the In-parallel chains (links) where the input and output links differ greatly in parallelism. In Four-bar chain, the input and output links will have the same structural parallelism with respect to the ground link and hence it is more suitable to generate less nonlinear functions.

\section{BASIS}

In view of the foregoing theory it is evident that the designer must have a tool to compare various distinct chains with the same number of links and d.o.f before making a final choice. Kinematic chains with dimensions (lengths) assigned on links must be compared for parallelism.

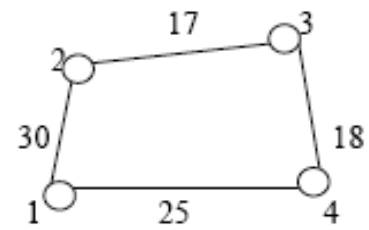

Fig. (3).

Fig. (3) shows a four bar chain with the links numbered from 1 to 4 while link dimensions are shown by $a=30, b=17$, $\mathrm{c}=18$ and $\mathrm{d}=25$. There is parallelism between links 1 and 3, and also between links 2 and 4 .

A four bar chain or a quadrangle is parallel dimensionally when all the sides are of equal lengths e.g. Fig. (1b). Also, it can be expected to be most rigid compared to any other combination of link lengths subjected to the condition that the sum of the link lengths is same.

It is of interest to know how much parallel are the links if the dimensions of the links assume different values, subjected to the condition, $\mathrm{a}+\mathrm{b}+\mathrm{c}+\mathrm{d}=\mathrm{k}$ (constant), where $\mathrm{a}, \mathrm{b}, \mathrm{c}, \mathrm{d}$ etc. are the link lengths.

For example, Fig. (2a) to Fig. (2e) shows quadrangles with link sizes in cms. They are the quadrangles with different link lengths, all having a sum of $60 \mathrm{~cm}$.

It is of interest to know how the dimensional parallelism is influenced by the change of dimensions, Fig. (2b) and Fig. (2d) and by keeping the identical link dimensions but changing their adjacency, Fig. (2d) and Fig. (2e).

To start with, parallelism $\mathrm{P}$ of a chain is expressed as a function of link dimensions ( $a, b$, c, etc.). The function is chosen in such a way that it satisfies one of the requirements of the most uniformly parallel chain. In Fig. (1b) all the dimensions are equal. One such function is:

$\mathrm{P}=\mathrm{a} * \mathrm{~b} * \mathrm{c} * \mathrm{~d}$

Subject to constraint,

$\mathrm{a}+\mathrm{b}+\mathrm{c}+\mathrm{d}=\mathrm{k}($ constant $)$

Thus, Eq (1) is also necessary but not fully sufficient to judge parallelism.

$P$ is maximum when, $a=b=c=d$ and for all other combinations the value of $\mathrm{P}$ is lower (See the proof below).

\subsection{Proof}

Maximize

$\mathrm{P}=$ a.b.c.d

Subject to the constraint

$\mathrm{a}+\mathrm{b}+\mathrm{c}+\mathrm{d}=\mathrm{k}$ (constant)

From eq. (2)

$a=k-b-c-d$ 
Substituting the above value of 'a' in eq. (1) we get

$\mathrm{P}=\mathrm{kbcd}-\mathrm{b}^{2} \mathrm{~cd}-\mathrm{c}^{2} \mathrm{bd}-\mathrm{d}^{2} \mathrm{bc}$

For $\mathrm{P}$ to be maximum, its partial derivatives with respect to $b, c$ and $d$ should be zero.

$$
\partial \mathrm{P} / \partial \mathrm{b}=\mathrm{kcd}-2 \mathrm{bcd}-\mathrm{c}^{2} \mathrm{~d}-2 \mathrm{bcd}=0
$$

Therefore,

$2 \mathrm{~b}+\mathrm{c}+\mathrm{d}=\mathrm{k}$

Similarly,

$\partial \mathrm{P} / \partial \mathrm{b}=0$, gives $2 \mathrm{c}+\mathrm{b}+\mathrm{d}=\mathrm{k}$

$\partial \mathrm{P} / \partial \mathrm{b}=0$, gives $2 \mathrm{~d}+\mathrm{b}+\mathrm{c}=\mathrm{k}$

Solving equations $\mathrm{a}, \mathrm{b}, \mathrm{c}$ simultaneously we get, $\mathrm{b}=\mathrm{c}=\mathrm{d}$

Using the above equations, in eq (1) and (a) we get,

$$
\mathrm{a}+3 \mathrm{~b}=\mathrm{k}
$$

and

$4 \mathrm{~b}=\mathrm{k}$

Therefore, $a=b$

Hence, $\mathrm{a}=\mathrm{b}=\mathrm{c}=\mathrm{d}$

It is yet to be proved that the resulting value of $\mathrm{P}$ is maximum. For this, the second derivatives of $\mathrm{P}$ must be negative i.e.

$\partial^{2} \mathrm{P} / \partial \mathrm{b}^{2}=-2 \mathrm{~cd}$ and same is the case with other derivatives.

Since

$$
\partial^{2} \mathrm{P} / \partial \mathrm{b}^{2} \text { is negative, } \mathrm{P} \text { is maximum }
$$

This can be verified from the examples, Fig. (2)

For Fig. (2a),

$$
\mathrm{P}=(15)^{4}=50625 \text {. }
$$

For Fig. (2b and c),

$$
\mathrm{P}=(20)^{2} \times(10)^{2}=40000 .
$$

For Fig. (2d and c),

$$
\mathrm{P}=10 \times 15 \times 17 \times 18=45900
$$

Higher value of $P$ in fact ensures near equal lengths of all the members of the chain. In other words, the rigidity of the links will be equal.

From the above values of $\mathrm{P}$, one can say that Chain Fig. (2a) having the highest value of $P$ is most uniformly parallel or rigid. $\mathrm{P}$ values of Chains Fig. (2b and $\mathbf{c})$ show that the links are less rigid/ parallel than Chain Fig. (2a). Chains Fig. (2d and e) are less uniform in rigidity than the Chain, Fig. (2a), but will be more rigid than the chains, Fig. (2b and c).

$P$ values of Chains Fig. (2b and $\mathbf{c}$ ) indicate that the extent of parallelism or rigidity present in both the configurations is same which is not true. Same is the case with Chains Fig. (2d and $\mathbf{c})$.

Geometric parallelism is more in the case of Fig. (2b) compared to that of Fig. (2c), but the $\mathrm{P}$ values violate this. Hence $\mathrm{P}$ alone cannot be taken as a measure of parallelism.
Strictly speaking, $\mathrm{P}$ is not a measure of parallelism as it ensures maximum value of the product ( $a$ b c d) when $\mathrm{a}=\mathrm{b}=\mathrm{c}=\mathrm{d}$, which happens to be one of the conditions for the parallelism of a four bar chain. Different values of $a, b$, c etc. is certainly an indication of different rigidities of these respective links, as rigidity depends upon many factors and link length is one amongst them. Thus the requirement of link adjacency for the links to become parallel must be included. This condition shall be sufficient to judge parallelism.

Obviously eqn. 1 and eqn. 2 cannot detect the extent of parallelism in different configurations, if the link dimensions are the same but their adjacencies are different. In order to deal with such cases,

(i) Adjacency of links must be taken into consideration and

(ii) Link lengths should be normalized.

The same is explained below:

\section{Adjacency of Links}

From the Fig. (1b) or (2a) it is clear that for two links, say 1 and 3 , to be parallel at all times, one of the requirements is links 2 and 4 should be of the same length. In other words the lengths of $a b$ and ad must be equal. Same is the case with the adjacencies of the other links. For example, considering link-2, its adjacent links are 1 and 3, hence $a b$ must be equal to bc. Thus for complete parallelism, $\mathrm{ab}=\mathrm{bc}=\mathrm{cd}=\mathrm{ad}$.

Thus for link-1,

$\mathrm{ab}-\mathrm{cd}=0$;

And link-2,

$\mathrm{ad}-\mathrm{bc}=0$;

In actual chains where the links are not of equal length, $(\mathrm{ab}-\mathrm{cd}) \neq 0$.

Thus, there is deviation $\epsilon_{1}=(a b-c d)$ for link-1. So is the case with the other links. Following the concept of least squares one can write a deviation function $\mathrm{Q}$ in the following manner.

$\mathrm{Q}=(\mathrm{ab}-\mathrm{ad})^{2}+(\mathrm{ab}-\mathrm{bc})^{2}+(\mathrm{bc}-\mathrm{cd})^{2}+(\mathrm{cd}-\mathrm{ad})^{2}$

$\mathrm{N}$

In general, $\quad \mathrm{Q}=\Sigma \epsilon^{2}$.

$$
\mathrm{i}=1
$$

where, $\epsilon_{i}$ is the deviation of the $i^{\text {th }}$ link and $n$ is the number of links in the chain.

For the most uniformly parallel chains such as that of Fig. (1a), $Q=0$ and for the other chains lesser value of $Q$ indicates greater uniformity in parallelism.

For example, consider the Figs. (2d and e).

For Fig. (2d), deviation of products for links 1,2,3 and 4 are respectively $36,120,20$ and 126 .

For Fig. (2e), the deviations are 90, 10, 85 and 75.

Substituting these values in eq.3 we find $Q$ for Fig. (2e) is lesser. 
Obviously, Fig. (2e) is more parallel than Fig. (2d); and similarly Fig. (2b) is more parallel than Fig. (2c).

\section{(ii) Normalization}

The foregoing theory is limited to the cases where the sum of all the link lengths is constant. In practice this may not hold as one has to compare the chains with different dimensions; not satisfying the constraint of eq. 2. For example consider the chain, (Fig. 3); the dimensions of the links are shown on the respective links in the figure in Centimeters.

Sum of all the link lengths is $90 \mathrm{cms}$, which is not the same as that of the chain Fig. (2d). Still one must be able to predict which of the chains, say Fig. (2d) or Fig. (3) is more parallel. In order to accomplish this, normalization of link lengths is proposed i.e. each link length is divided by the sum of the link lengths of the concerned chain. This indirectly satisfies the constraint of eq. 2. Since the sum of all the normalized lengths of a chain is 1 . Application of eq. 1 then leads to the comparison.

For Fig. (2d), normalized link lengths are 1/60, 1/60, $17 / 60,18 / 60$.

Eq. 1 gives:

$$
\mathrm{P}=15 / 60 \times 10 / 60 \times 17 / 60 \times 18 / 60=0.00354
$$

For Fig. (3), the normalized lengths are 25/90, 30/90, $17 / 90,18 / 90$

Hence,

$$
\mathrm{P}=25 / 90 \times 30 / 90 \times 17 / 90 \times 18 / 90=0.0349
$$

Value of $\mathrm{P}$ being higher in the case of Fig. (2d), its links are more uniformly rigid than the links of the chain, (Fig. 3).

\section{APPLICATION TO MULTI-LOOP CHAINS}

Comparison is possible only among Kinematic chains with the same number of links and d.o.f. These chains usually consist of high connectivity links such as ternary, quaternary etc. but the number of design parameters will be the same. When computing the sum of link lengths, lengths of all sides of polygonal links should be included.

If two chains with different link dimensions are to be compared, the product of the normalized dimensions of all the links of a chain needs to be considered. The links of the chain with higher product will be more uniformly rigid.

On the other hand, if two chains have identical links i.e. a particular link assortment (dimensional links) are arranged in two or more different ways structurally, then eq. 3 needs to be applied. The products should, however be computed systematically for every dimension of the chain going round the loop in which the particular dimension (side of the link) lies. This should include not only the clear inner loops but also the peripheral loop of the chains. For illustration consider two seven link, single d.o.f. chains which can be adopted as manipulator structures, as shown in Fig. (4a and b).

Links are numbered 1, 2, 3, etc inside the fig. while the link lengths in cms are marked on the periphery. Loops are indicated by encircled numbers (1), (2). Outside or peripheral loop is always the last loop, if numbered or not numbered but should be taken as loop (3). Dimensions taken are purely arbitrary.

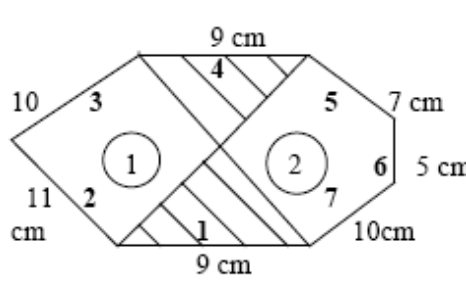

(a)

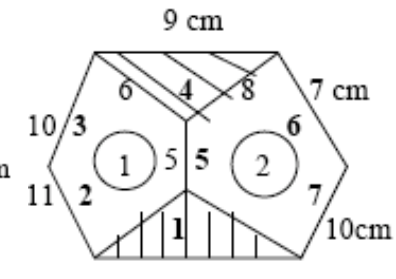

$9 \mathrm{~cm}$
Fig. (4). Seven Link single d.o.f chains.

The product of the adjacent link for every loop of each are computed.

For chain Fig. (4a):

LOOP 1:-

\begin{tabular}{|c|c|c|c|}
\hline Links & $\underline{\text { Product }}$ & $\underline{\text { Links }}$ & Products \\
\hline 1 and 2 & 66 & 1 and 4 & 56 \\
\hline 2 and 3 & 110 & 4 and 5 & 56 \\
\hline 3 and 4 & 60 & 5 and 6 & 35 \\
\hline nd 1 & 36 & 6 and 7 & 50 \\
\hline
\end{tabular}

LOOP 2:-

7 and $1 \quad 70$

LOOP 3:-

$\begin{array}{cc}\underline{\text { Links }} & \text { Product } \\ 1 \text { and } 2 & 99 \\ 2 \text { and } 3 & 110 \\ 3 \text { and } 4 & 90 \\ 4 \text { and } 5 & 63 \\ 5 \text { and } 6 & 35 \\ 6 \text { and } 7 & 50 \\ 7 \text { and } 1 & 90\end{array}$

Deviation of the products, say for link-1, loop (1) is given by the difference in the products of links ( 1 and 2) and links (4 and 1), as link-1 is common between these two. In this case it is equal to $(66-36)=30$. Likewise deviation can be obtained for every link in the corresponding loop and then application of eq. 3 leads to total deviation of the chain. Thus in this case total deviation is 11214 .

Similarly proceeding, for chain Fig. (4b) the products are given below:

LOOP 1:-

$\begin{array}{cc}\underline{\text { Links }} & \text { Product } \\ 1 \text { and } 2 & 66 \\ 2 \text { and } 3 & 110 \\ 3 \text { and } 4 & 60 \\ 4 \text { and } 5 & 30 \\ 5 \text { and } 1 & 30\end{array}$




\section{LOOP 2:-}

\begin{tabular}{|c|c|}
\hline Links & Product \\
\hline 1 and 5 & 35 \\
\hline 5 and 4 & 40 \\
\hline 4 and 6 & 56 \\
\hline 6 and 7 & 70 \\
\hline 7 and 1 & 70 \\
\hline
\end{tabular}

LOOP 3:-

$\begin{array}{cc}\underline{\text { Links }} & \text { Product } \\ 1 \text { and } 2 & 99 \\ 2 \text { and } 3 & 110 \\ 3 \text { and } 4 & 90 \\ 4 \text { and } 6 & 63 \\ 6 \text { and } 7 & 70 \\ 7 \text { and } 1 & 70\end{array}$

Total deviation in this case is 9538 .

Comparison of the deviation of the products shows that it is less in case of Fig. (4b) and hence it is dimensionally more parallel. Working out in the above manner all the chains with a specified number of the links and d.o.f can be easily compared.

\section{CONCLUSION}

As we are aware that More parallel chains are considered as more rigid and Less parallel chains are more suitable for generation of greater workspace. The following conclusions from the above methodology can be added to these and utilized beneficially:
1) The product of the lengths of all the links is the criterion to judge the equality of link lengths; higher the product, greater is the rigidity of the links. This is only a necessary condition but not sufficient to judge parallelism.

2) Deviation of the products of the adjacent links is another criterion. Smaller the deviation, greater is the parallelism. Thus eqs. 1 and 3 form necessary and sufficient conditions to judge the parallelism.

3) While working out the products, independent loops and the peripheral loop should be considered. This ensures that the product for each dimension, in a multi loop chain, is taken.

\section{REFERENCES}

[1] K.H. Hunt, "Structural Kinematics of in parallel actuated Robot Arms", ASME Journal of Mechanism, Transmission and Automation in design, vol. 105, no. 4, pp. 705-712, 1983.

[2] G.R. Pennock and D.I. Dassner, "Kinematic analysis of a planar eight bar linkage-application to a platform type robot", ASME Journal of Mechanical Design, vol. 144, pp. 87-95, 1992.

[3] C.V. Parenti and C. Innocenti, "Forward displacement analysis of parallel mechanisms; closed form solution of PPR3S structures", ASME Journal of Mechanical Design, vol. 144, pp. 68-72, 1992.

[4] A.C. Rao, "Platform-type planar robots; topology based selection for rigidity and workspace", Journal Of Robotic Systems, vol. 14, no. 5, pp. 355-364, 1997.

[5] A.C. Rao and A. Jagadesh, "Topology based characteristics of kinematic chains: workspace, rigidity, input joints and isomorphism", Mechanism and Machine Theory, vol. 33, pp. 625638, 1998.

[6] A.C. Rao, "Topological characteristics of linkages mechanisms with particular references to platform type-robots", Mechanism and Machine Theory, vol. 30, pp. 33-42, 1995.

[7] A. Srinath and A.C. Rao, "Kinematic chains for robot hands: grasp and rigidity", Mechanism and Machine Theory, vol. 42, pp. 691$697,2007$.

[8] A.C. Rao and A. Srinath, "Planar linkages: structural influence on mechanical advantage and function generation", vol. 42, pp. 472$481,2007$. 